\title{
Electrospinning Preparation and Photocatalytic Activity of Porous $\mathrm{TiO}_{2}$ Nanofibers
}

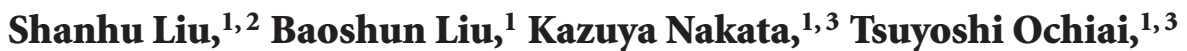 \\ Taketoshi Murakami, ${ }^{1}$ and Akira Fujishima ${ }^{1,3}$ \\ ${ }^{1}$ Photocatalyst Group, Kanagawa Academy of Science and Technology, KSP East 412, 3-2-1 Sakado, Takatsu-ku, Kawasaki, \\ Kanagawa 213-0012, Japan \\ ${ }^{2}$ Institute of Environmental and Analytical Sciences, College of Chemistry and Chemical Engineering, Henan University, \\ Kaifeng 475001, China \\ ${ }^{3}$ Research Institute for Science and Technology, Energy and Environment, Photocatalyst Research Division, \\ Tokyo University of Science, 1-3 Kagurazaka, Shinjuku-ku, Tokyo 162-8601, Japan
}

Correspondence should be addressed to Kazuya Nakata, pg-nakata@newkast.or.jp

Received 14 January 2012; Accepted 20 April 2012

Academic Editor: Gajanan S. Bhat

Copyright (c) 2012 Shanhu Liu et al. This is an open access article distributed under the Creative Commons Attribution License, which permits unrestricted use, distribution, and reproduction in any medium, provided the original work is properly cited.

\begin{abstract}
Porous $\mathrm{TiO}_{2}$ nanofibers were prepared via a facile electrospinning method. The carbon nanospheres were mixed with the ethanol solution containing both poly(vinylpyrrolidone) and titanium tetraisopropoxide for electrospinning; and subsequent calcination of as-spun nanofibers led to thermal decomposition of carbon nanospheres, leaving behind pores in the $\mathrm{TiO}_{2}$ nanofibers. The morphology and phase structure of the products were investigated with scanning electron microscopy (SEM), transmission electron microscopy (TEM), and X-ray diffraction (XRD). Furthermore, the photocatalytic activity of porous $\mathrm{TiO}_{2}$ nanofibers was evaluated by photodecomposition of methylene blue under UV light. Results showed that the porous $\mathrm{TiO}_{2}$ nanofibers have higher surface area and enhanced photocatalysis activity, compared to nonporous $\mathrm{TiO}_{2}$ nanofibers.
\end{abstract}

\section{Introduction}

$\mathrm{TiO}_{2}$-based photocatalysts have been one of the most active areas in heterogeneous catalysis due to their great catalytic abilities for removing environmental pollutants relating to waste water, polluted air, and spilling water $[1,2]$. Over the past few years, it has been demonstrated that the photocatalytic activity strongly depends on the specific surface area of photoactive materials [3-5]. Many strategies have been developed to fabricate porous and hollow $\mathrm{TiO}_{2}$ nanostructures to enhance their photocatalytic performance [6-10]. Among these methods for generating porous nanostructures, especially for porous nanofibers, electrospinning is a well-established top-down approach [11-15]. For instance, $\mathrm{Li}$ et al. synthesized porous $\mathrm{TiO}_{2}$-based nanofibers via a combined electrospinning and alkali dissolution method; they firstly prepared $\mathrm{TiO}_{2} / \mathrm{SiO}_{2}$ composite nanofibers by electrospinning and sintering and then leached out silica with alkaline solution to produce porous $\mathrm{TiO}_{2}$ microstructures [16]. Mesoporous $\mathrm{TiO}_{2}$ nanofibers were prepared by electrospinning $\mathrm{TiO}_{2}$-nanoparticle-containing polymeric spinning solution [17]. Furthermore, in our previous work, $\mathrm{TiO}_{2}$ fibers with interior hollow channels were fabricated via multifluidic electrospinning with the obvious improvement of photocatalytic activity [18]. In this paper, porous $\mathrm{TiO}_{2}$ nanofibers were fabricated through an electrospinning method with carbon nanospheres as sacrificial template. Firstly, the spinning solution containing carbon nanospheres was prepared. Our aim was to retain the carbon nanospheres in the as-spun nanofibers, followed by calcination leading to the thermal decomposition of carbon nanospheres, leaving behind a porous $\mathrm{TiO}_{2}$ nanofiber structure.

\section{Experimental}

2.1. Preparation of Carbon Nanospheres. Carbon nanospheres were synthesized via a hydrothermal method by 


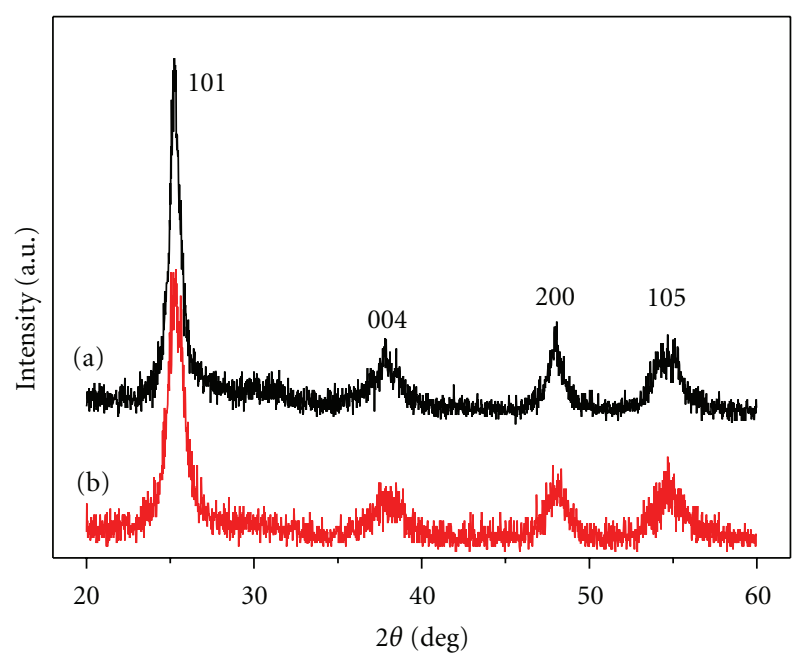

Figure 1: XRD patterns of $\mathrm{TiO}_{2}$ nanofibers (a) and $\mathrm{P}-\mathrm{TiO}_{2}$ nanofibers (b).

using glucose as reagents as previously reported [19]. In brief, $3.5 \mathrm{~g}$ glucose (Wako, Japan) was dissolved in $40 \mathrm{~mL}$ of water to form a clear solution, which was placed in a teflonsealed autoclave $(50 \mathrm{~mL})$ and maintained at $170^{\circ} \mathrm{C}$ for $5 \mathrm{~h}$. The black or puce products were isolated by centrifugation, washed several times under sonication with water and ethanol, then oven-dried at $60^{\circ} \mathrm{C}$ for characterization and subsequent application.

2.2. Preparation of Porous $\mathrm{TiO}_{2}$ Nanofibers. In the typical electrospinning procedure, $0.3 \mathrm{~g}$ of carbon nanospheres was dissolved in $10 \mathrm{~mL}$ of ethanol to form a homogeneous solution, followed by adding $1.0 \mathrm{~g}$ of polyvinylpyrrolidone (PVP, Mw $=130,000$, Aldrich) and $3 \mathrm{~mL}$ of acetic acid (Wako, Japan). After stirring for one hour, this solution was added to $1.0 \mathrm{~mL}$ of titanium tetraisopropoxide ( $\mathrm{Ti}(\mathrm{OiPr})_{4}$, Aldrich) (Wako, Japan) and stirred for another hour. The mixed solution was fed through a nozzle at a feeding rate of $4 \mathrm{~mL} \mathrm{~min}^{-1}$. The applied voltage was $22 \mathrm{kV}$ and the working distance was $15 \mathrm{~cm}$. After electrospinning process, the obtained fibers were calcined in air at $500^{\circ} \mathrm{C}$ for $2 \mathrm{~h}$ at a heating rate of $5^{\circ} \mathrm{C} \mathrm{min}{ }^{-1}$ to form porous $\mathrm{TiO}_{2}$ nanofibers, which were denoted as $\mathrm{P}-\mathrm{TiO}_{2}$ nanofibers. For comparison, we have prepared $\mathrm{TiO}_{2}$ nanofibers in the absence of carbon nanospheres, and the rest of the synthesis conditions were kept the same as those described above in the typical experiment.

2.3. Characterization. The morphology of the nanofibers was examined using scanning electron microscopy (JSM5400, JEOL, Japan). X-ray diffraction (XRD) patterns were recorded using a Rigaku RINT 1500 diffractometer with the use of $\mathrm{Cu} \mathrm{K} \alpha$ radiation. Nitrogen adsorption isotherms were measured at $-196^{\circ} \mathrm{C}$ using a Micromeritics system (Gemini V, Shimadzu, Japan). UV-vis absorption was performed on UV-1700 (Shimadzu, Japan) spectrophotometer.

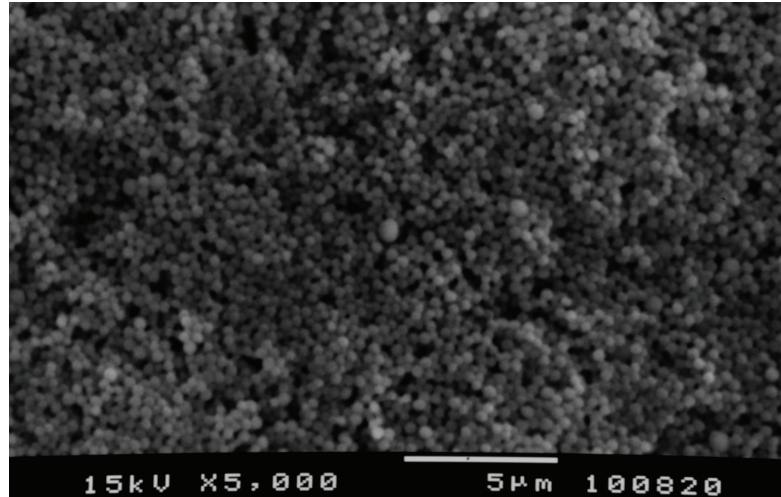

(a)

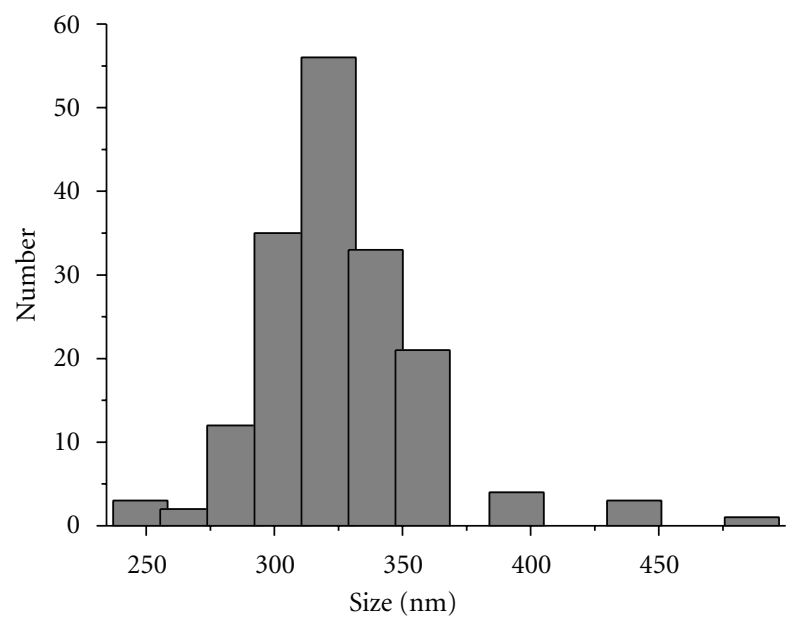

(b)

FIGURE 2: SEM image (a) and the corresponding histogram of the diameters (b) of carbon nanospheres.

2.4. Photocatalysis Measurement. The photocatalytic activity of these nanofibers was evaluated as their ability to decompose the aqueous suspension of methylene blue (MB) under UV-light at room temperature. Prior to the light irradiation, $20 \mathrm{~mL}$ of $\mathrm{MB}$ solution $\left(20 \mathrm{mg} \mathrm{mL}^{-1}\right)$ was mixed with the catalyst $(20 \mathrm{mg}$ ) and kept in the dark for 200 minutes to establish an adsorption-desorption equilibrium. The concentration of dye solution was measured with UV irradiation time by using a UV-vis spectrometer. To monitor the change in concentration of $\mathrm{MB}$, the absorbance spectra of $\mathrm{MB}$ were recorded at its maximum absorption.

\section{Results and Discussion}

The crystalline structure and phase composition of the products were characterized by XRD. As shown in Figure 1, all of the obtained products matched well with the standard pattern of anatase (JCPDS no. 21-1272), with four reflections at $25.3,37.9,48.1$, and 54.2, corresponding to (101), (004), (200), and (105), respectively. More importantly, no impurity peaks were observed in the XRD patterns of $\mathrm{P}_{-} \mathrm{TiO}_{2}$ 


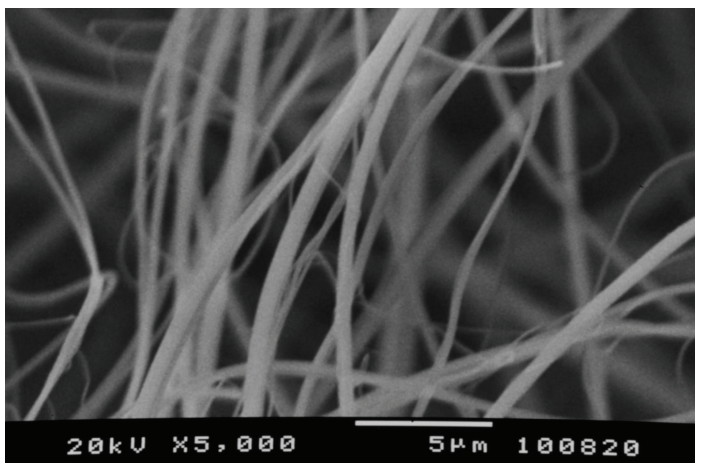

(a)

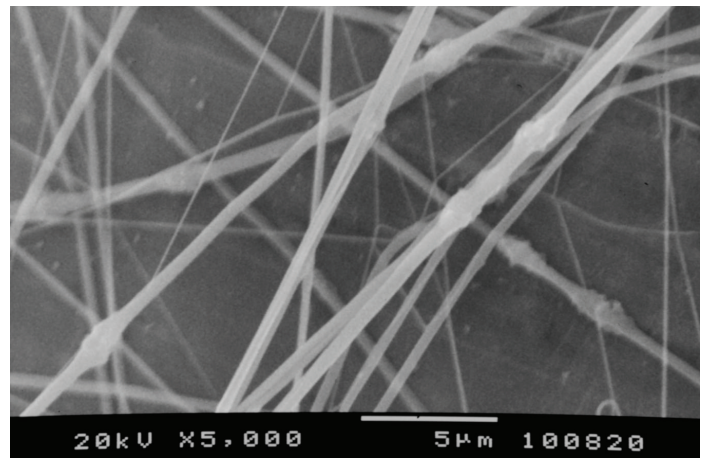

(c)

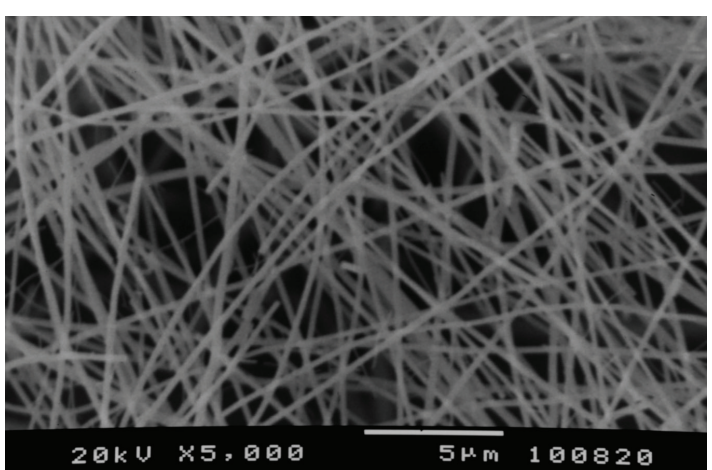

(b)

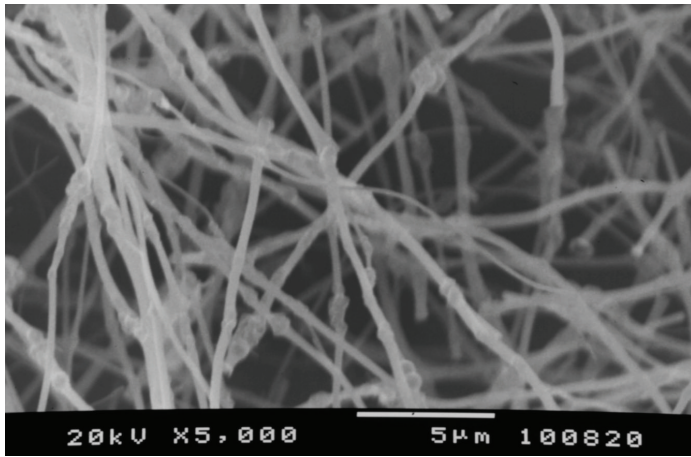

(d)

FIGURE 3: SEM image of nanofibers prepared without $(a, b)$ and with carbon nanospheres ( $c, d)$; SEM images of nanofibers before $(a, c)$ and after (b, d) calcination.

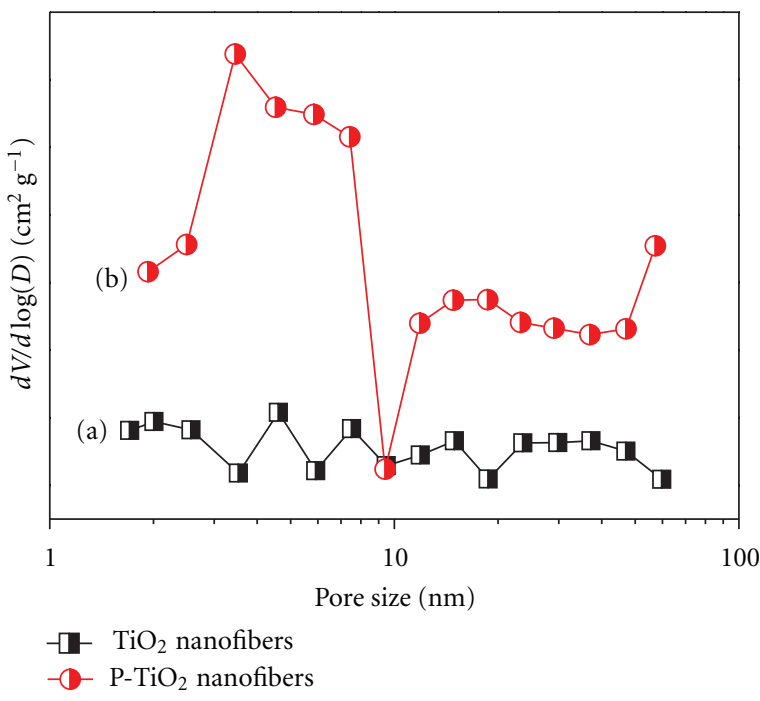

Figure 4: Pore size distributions of the $\mathrm{TiO}_{2}$ nanofibers (a) and P$\mathrm{TiO}_{2}$ nanofibers (b).

nanofibers, suggesting that the crystal phase is not changed during the process of generating pores.

Carbon nanospheres were investigated by SEM analyses. Figure 2 represents SEM image and the corresponding histogram of the diameters of carbon nanospheres. The nanospheres are monodispersed and uniform on a large

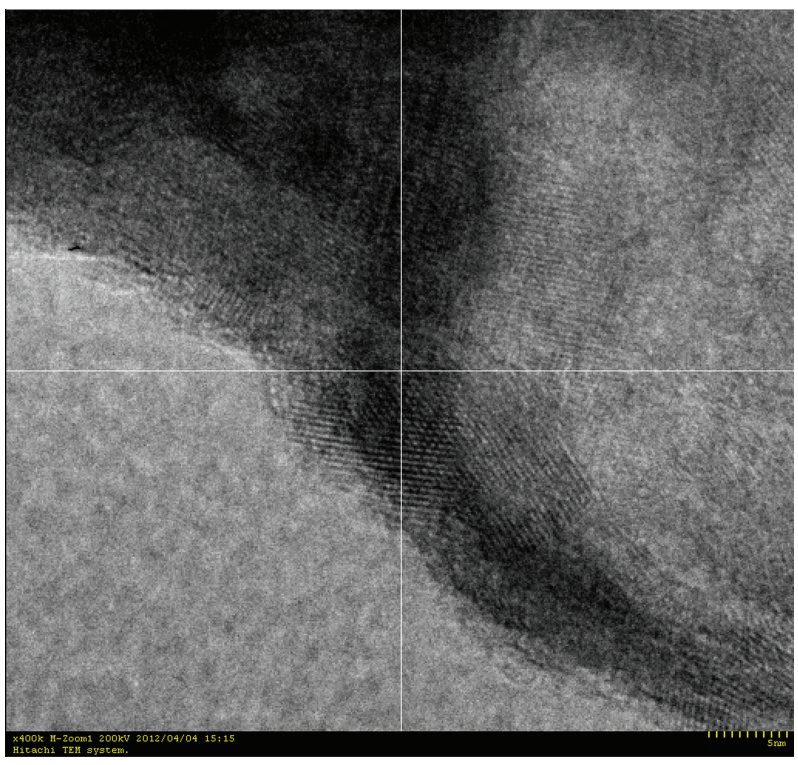

FIgure 5: HRTEM image of $\mathrm{P}_{-} \mathrm{TiO}_{2}$ nanofibers.

scale. A statistical analysis yields an average size of $320 \mathrm{~nm}$ in diameter. In this paper, carbon nanospheres could be removed by calcination simultaneously during the process of removing PVP, without needing an additional removing 


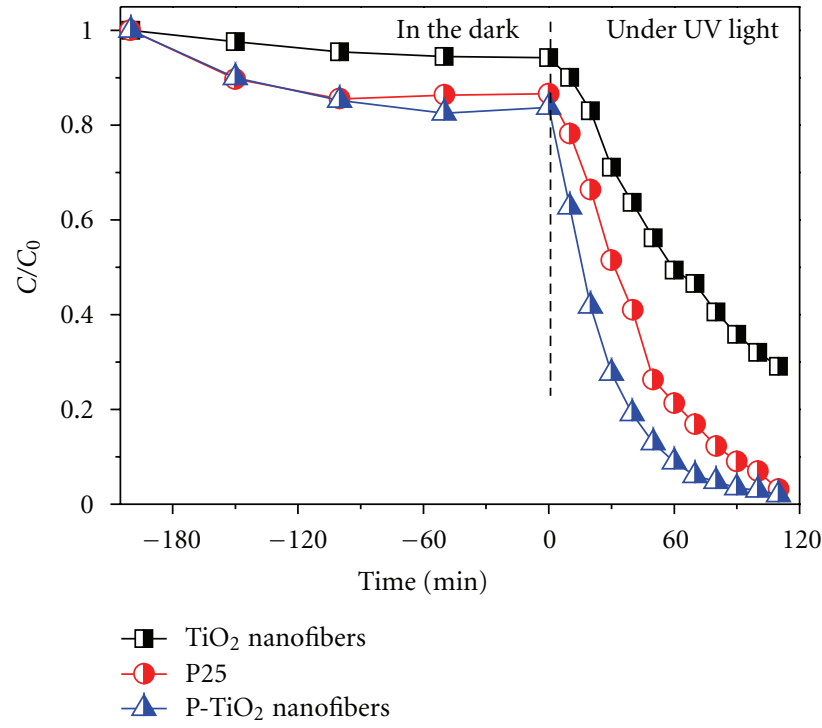

(a)

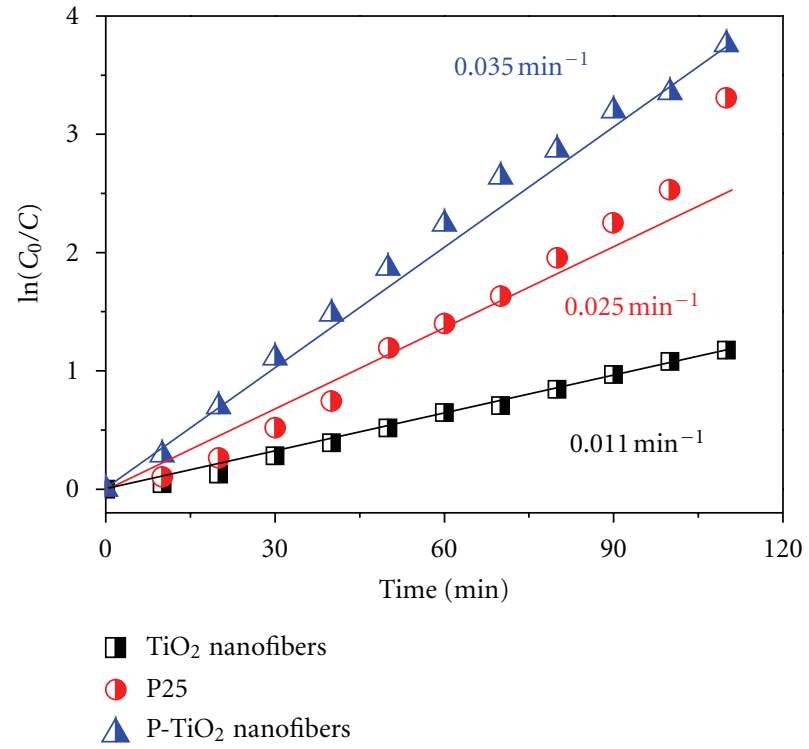

(b)

Figure 6: Liquid-phase photocatalytic degradation (a) and photocatalytic degradation reaction kinetics (b) of $\mathrm{MB}$ against TiO ${ }_{2}$ nanofibers, $\mathrm{TiO}_{2} \mathrm{P} 25$, and $\mathrm{P}-\mathrm{TiO}_{2}$ nanofibers.

step. Moreover, during the calcination carbon nanospheres only release nontoxic $\mathrm{CO}_{2}$ and $\mathrm{H}_{2} \mathrm{O}$ but not toxic gases.

Figure 3 shows SEM images of $\mathrm{TiO}_{2}$ nanofibers and $\mathrm{P}$ $\mathrm{TiO}_{2}$ nanofibers before and after calcination. It was observed that the surface of nanofibers without carbon nanospheres was smooth (Figure 3(a)) while the surface of nanofibers with carbon nanospheres was lumpy at places where the nanospheres were present (Figure 3(c)). After calcination, the average diameters of the nanofibers decreased slightly (Figures 3(b) and 3(d)). This is because the organic components (PVP or carbon nanospheres) were removed by combustion and the inorganic component had crystallized. The carbon nanospheres were removed via calcination leaving behind pores in the nanofibers. The diameters of nonporous $\mathrm{TiO}_{2}$ nanofibers and $\mathrm{P}-\mathrm{TiO}_{2}$ nanofibers were statistically determined to be 230 and $330 \mathrm{~nm}$, respectively.

The porous structure within the $\mathrm{P}_{-} \mathrm{TiO}_{2}$ nanofibers was further confirmed by the $\mathrm{N}_{2}$ physisorption experiments. Despite its larger diameter, $\mathrm{P}_{-} \mathrm{TiO}_{2}$ nanofibers have larger specific surface area $\left(33 \mathrm{~m}^{2} / \mathrm{g}\right)$ than $\mathrm{TiO}_{2}$ nanofibers $\left(9 \mathrm{~m}^{2} / \mathrm{g}\right)$. As shown in Figure 4 , the pores volume of $\mathrm{P}-$ $\mathrm{TiO}_{2}$ nanofibers was larger than that of nonporous $\mathrm{TiO}_{2}$ nanofibers. However, the $\mathrm{P}-\mathrm{TiO}_{2}$ nanofibers possess wide pore size distributions from several nanometers to tens of nanometers because of the complex interplay between the shrink of the nanofibers and the decomposition of organics during calcination.

More evidence on crystallinity of $\mathrm{P}-\mathrm{TiO}_{2}$ nanofibers was obtained from the TEM analysis. As shown in Figure 5, the distinct contrast derived from the difference of electron density in TEM image further confirms the porous structure of the nanofibers. The crystal lattice fringes indicates that the nanofibers are formed by geometrically random $\mathrm{TiO}_{2}$ nanograins, which exhibit high crystallinity after calcination.

To determine the photocatalytic activity of $\mathrm{P}^{-\mathrm{TiO}_{2}}$ nanofibers, the degradation experiments of $\mathrm{MB}$ against $\mathrm{P}_{-} \mathrm{TiO}_{2}$ nanofibers, $\mathrm{TiO}_{2}$ P25 (AEROXIDE), and $\mathrm{TiO}_{2}$ nanofibers were carried out. In the dark $(-200 \sim 0 \mathrm{~min})$, the relative concentration of $\mathrm{MB}$ in the solution is slightly decreased owing to the adsorption of $\mathrm{MB}$ on the catalysts; the concentration decrease is slightly greater for $\mathrm{P}-\mathrm{TiO}_{2}$ nanofibers than for $\mathrm{TiO}_{2}$ nanofibers, which reflects that the surface area is greater for $\mathrm{P}-\mathrm{TiO}_{2}$ nanofibers. As shown in Figure $6(\mathrm{a}), 90 \%$ of $\mathrm{MB}$ could be decomposed by $\mathrm{P}-\mathrm{TiO}_{2}$ nanofibers within $60 \mathrm{~min}$ and was degraded completely after 110 min. Although the most of MB was also decomposed in the presence of the P25 after the same period, the photocatalytic activity was lower than $\mathrm{P}_{-} \mathrm{TiO}_{2}$ nanofibers. In contrast, the photocatalytic activity of $\mathrm{TiO}_{2}$ nanofibers was lowest; about $50 \% \mathrm{MB}$ remained after $60 \mathrm{~min}$ irradiation. The reaction rate constant of the $\mathrm{MB}$ degradation against $\mathrm{P}$ $\mathrm{TiO}_{2}$ nanofibers $\left(0.035 \mathrm{~min}^{-1}\right)$ is three times higher than that of $\mathrm{TiO}_{2}$ nanofibers $\left(0.011 \mathrm{~min}^{-1}\right)$ (Figure 6(b)). The results indicate that $\mathrm{P}-\mathrm{TiO}_{2}$ nanofibers have greater photocatalytic activity than nonporous $\mathrm{TiO}_{2}$ nanofibers, suggesting that the surface area is an important factor for adsorption and catalysis.

\section{Conclusion}

In conclusion, porous $\mathrm{TiO}_{2}$ nanofibers were prepared via an efficient and simple electrospinning method by using carbon nanospheres as sacrificial template. Carbon nanospheres together with PVP could be removed at the same time 
during calcination. Microstructural analysis and properties evaluation revealed that porous $\mathrm{TiO}_{2}$ nanofibers have higher surface area and enhanced photocatalytic activity compared to nonporous $\mathrm{TiO}_{2}$ nanofibers. In addition, the method for synthesis of porous $\mathrm{TiO}_{2}$ nanofibers presented here can be extended to synthesize other one-dimensional porous nanostructures.

\section{Acknowledgments}

This work was supported by a Grant-in-Aid for Scientific Research (B) from the Ministry of Education, Culture, Sports, Science and Technology of Japan and National Natural Science Foundation of China (21105021).

\section{References}

[1] A. Fujishima and K. Honda, "Electrochemical photolysis of water at a semiconductor electrode," Nature, vol. 238, no. 5358, pp. 37-38, 1972.

[2] R. Wang, K. Hashimoto, A. Fujishima et al., "Light-induced amphiphilic surfaces," Nature, vol. 388, no. 6641, pp. 431-432, 1997.

[3] S. Lee, K. Lee, G. D. Moon et al., "Preparation of macroporous carbon nanofibers with macroscopic openings in the surfaces and their applications," Nanotechnology, vol. 20, no. 44, Article ID 445702, 2009.

[4] K. Brezesinski, R. Ostermann, P. Hartmann, J. Perlich, and T. Brezesinski, "Exceptional photocatalytic activity of ordered mesoporous $\beta-\mathrm{Bi}_{2} \mathrm{O}_{3}$ thin films and electrospun nanofiber mats," Chemistry of Materials, vol. 22, no. 10, pp. 3079-3085, 2010.

[5] G. Cappelletti, S. Ardizzone, F. Spadavecchia, D. Meroni, and I. Biraghi, "Mesoporous titania nanocrystals by hydrothermal template growth," Journal of Nanomaterials, vol. 2011, Article ID 597954, 9 pages, 2011.

[6] B. Liu, K. Nakata, M. Sakai et al., "Mesoporous $\mathrm{TiO}_{2}$ core-shell spheres composed of nanocrystals with exposed high-energy facets: facile synthesis and formation mechanism," Langmuir, vol. 27, no. 13, pp. 8500-8508, 2011.

[7] J.-Y. Shin, D. Samuelis, and J. Maier, "Sustained lithiumstorage performance of hierarchical, nanoporous anatase $\mathrm{TiO}_{2}$ at high rates: emphasis on interfacial storage phenomena," Advanced Functional Materials, vol. 21, no. 18, pp. 3464-3472, 2011.

[8] F. Iskandar, A. B. D. Nandiyanto, K. M. Yun, C. J. Hogan, K. Okuyama, and P. Biswas, "Enhanced photocatalytic performance of brookite $\mathrm{TiO}_{2}$ macroporous particles prepared by spray drying with colloidal templating," Advanced Materials, vol. 19, no. 10, pp. 1408-1412, 2007.

[9] H. Ming, Z. Ma, H. Huang et al., "Nanoporous $\mathrm{TiO}_{2}$ spheres with narrow pore size distribution and improved visible light photocatalytic abilities," Chemical Communications, vol. 47, no. 28, pp. 8025-8027, 2011.

[10] K. Nakata, B. Liu, Y. Ishikawa et al., "Fabrication and photocatalytic properties of $\mathrm{TiO}_{2}$ nanotube arrays modified with phosphate," Chemistry Letters, vol. 40, no. 10, pp. 1107-1109, 2011.

[11] D. Li and Y. Xia, "Fabrication of titania nanofibers by electrospinning," Nano Letters, vol. 3, no. 4, pp. 555-560, 2003.
[12] S. Madhugiri, B. Sun, P. G. Smirniotis, J. P. Ferraris, and K. J. Balkus, "Electrospun mesoporous titanium dioxide fibers," Microporous and Mesoporous Materials, vol. 69, no. 1-2, pp. 77-83, 2004.

[13] S. Cavaliere, S. Subianto, I. Savych, D. J. Jones, and J. Rozière, "Electrospinning: designed architectures for energy conversion and storage devices," Energy and Environmental Science, vol. 4, no. 12, pp. 4761-4785, 2011.

[14] L. Ji, C. Saquing, S. A. Khan, and X. Zhang, "Preparation and characterization of silica nanoparticulate- polyacrylonitrile composite and porous nanofibers," Nanotechnology, vol. 19, no. 8, Article ID 085605, 2008.

[15] S. Ramkumar, U. Sata, and M. Hussain, "Personal protective fabric technologies for Chemical Countermeasures," in Advances in Biological and Chemical Terrorism Countermeasures, chapter 8, pp. 203-227, Taylor and Francis Group, 2008.

[16] Q. Li, D. Sun, and H. Kim, "Fabrication of porous $\mathrm{TiO}_{2}$ nanofiber and its photocatalytic activity," Materials Research Bulletin, 2011.

[17] C. Wessel, R. Ostermann, R. Dersch, and B. M. Smarsly, "Formation of inorganic nanofibers from preformed $\mathrm{TiO}_{2}$ nanoparticles via electrospinning," Journal of Physical Chemistry C, vol. 115, no. 2, pp. 362-372, 2011.

[18] T. Zhao, Z. Liu, K. Nakata et al., "Multichannel $\mathrm{TiO}_{2}$ hollow fibers with enhanced photocatalytic activity," Journal of Materials Chemistry, vol. 20, no. 24, pp. 5095-5099, 2010.

[19] X. Sun and Y. Li, "Colloidal carbon spheres and their core/ shell structures with noble-metal nanoparticles," Angewandte Chemie, vol. 43, no. 5, pp. 597-601, 2004. 

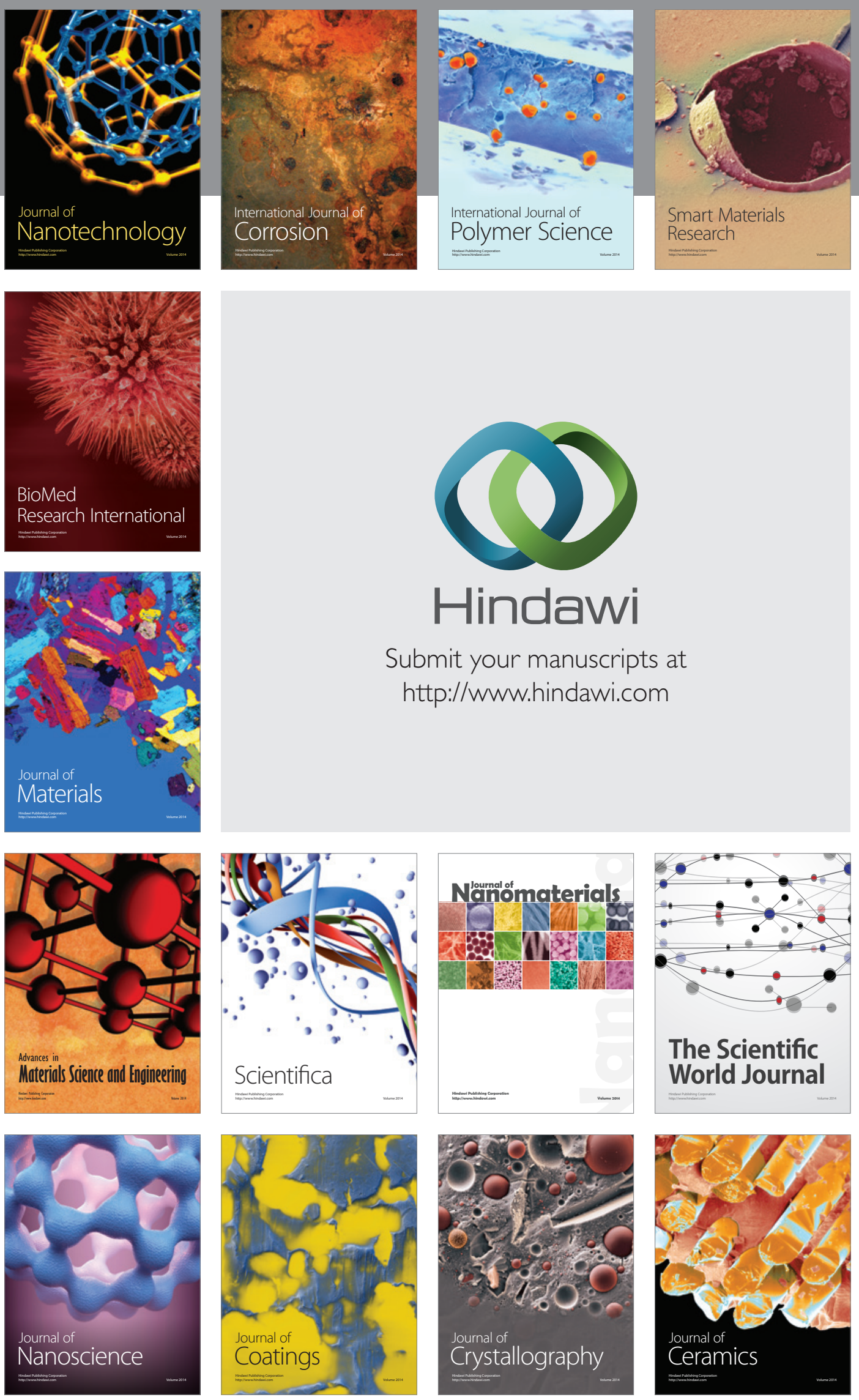

The Scientific World Journal

Submit your manuscripts at

http://www.hindawi.com

\section{World Journal}

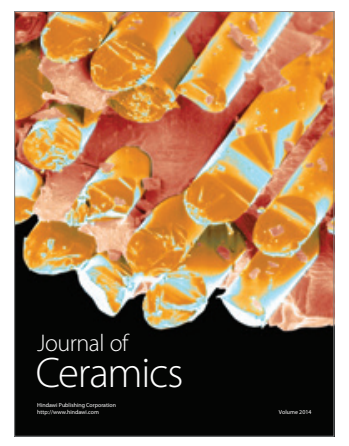

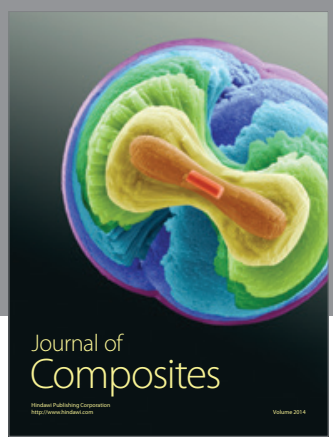
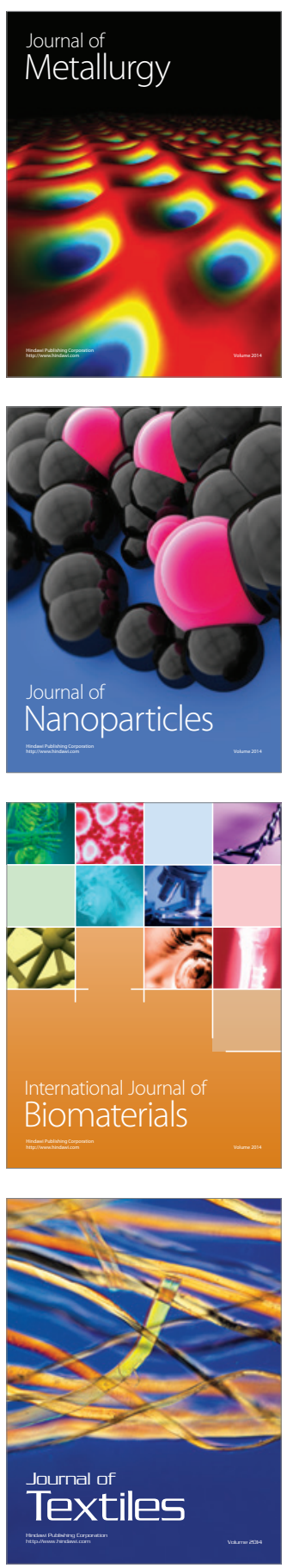